\title{
Pesquisa Exploratória Comparativa entre Artigos e Patentes Sobre Maturidade (Prontidão) Tecnológica
}

\section{Comparative Exploratory Research Between Articles and Patents on Technological Readiness}

\author{
André Góes Paternostro ${ }^{1,2}$ \\ Cristina M. Quintella ${ }^{1}$ \\ Handerson Jorge Dourado Leite ${ }^{3,4}$ \\ ${ }^{1}$ Universidade Federal da Bahia, Salvador, BA, Brasil \\ ${ }^{2} \mathrm{CDM}$ Group, Salvador, BA, Brasil \\ ${ }^{3}$ Instituto Federal de Educação, Ciência e Tecnologia da Bahia, Salvador, BA, Brasil \\ ${ }^{4}$ Fundação de Amparo à Pesquisa do Estado da Bahia, Salvador, BA, Brasil
}

\begin{abstract}
Resumo
Neste artigo apresenta-se uma análise comparativa exploratória com o propósito de identificar os principais atores em conceitos de maturidade tecnológica. Inicialmente foi realizada uma análise comparativa nas bases de artigos (Web of Science, Scopus, Periódicos Capes e Google Academics) e de patentes (Derwent, Orbit, WIPO - Patentscope, Spacenet e PatenteInspiration). A classificação TRL - nível de maturidade (prontidão) tecnológica foi a mais encontrada. Para uma análise mais detalhada da TRL, foi escolhida a base Scopus (2.787 artigos) e Espacenet, usando o software Questel Orbit (110 patentes). Descobriu-se que os artigos começaram a surgir em 1991, e as patentes, em 1998. As áreas tecnológicas dominantes dos artigos são Engenharia (1.947) e Ciência da Computação (540), e das patentes foi G06 (70) de simuladores, métodos de computar condições existentes ou previstas em um sistema ou dispositivo real. O país que se destaca pelo número de artigos (1.307) e patentes (48) é os Estados Unidos. As empresas de maior relevância estão concentradas no setor aeroespacial (NASA, Raytheon, Boeing, Safran Thales Group).
\end{abstract}

Palavras-chave: Nível de Maturidade Tecnológica (NMT-TRL). Nível de Maturidade para Manufatura (NMR). Calculadora de Maturidade Tecnológica.

\begin{abstract}
This article performed a comparative exploratory analysis in order to identify the main actors in concepts of technological readiness level. For this, an initial comparative analysis was carried out in the databases of articles (Web of Science, Scopus, Capes Newspapers, Google Academics) and patents (Derwent, Orbit, WIPO - Patentscope, Spacenet and PatentInspiration). The TRL - level of technological maturity (readiness) was the most widely found. For TRL detailed analysis were chosen the databasis Scopus (2,787 articles) and Espacenet using the software Questel Orbit (110 patents). The articles began in 1991 and the patents in 1998. The articles technology areas that stood out were engineering $(1,947)$ and computer science $(540)$, and for patents was G06 (70) simulators, methods of computing existing or predicted conditions in a real system or device. The country that stands out both in number of articles $(1,307)$ and patents $(48)$ is United States. The most relevant companies are from the aerospace sector (NASA, Raytheon, Boeing, Safran Thales Group).
\end{abstract}

Keywords: Technology Readiness Level (TRL). Manufacturing Readiness Level (MRL). Technological Readiness Level Calculator. Technology Readiness.

Área Tecnológica: Gestão de CTI. Inovação. 


\section{Introdução}

A criação de diferentes níveis de Maturidade Tecnológica foi uma construção crescente dentro da NASA desde o final da década de 1960, motivada pela necessidade de articular o status de uma nova tecnologia para seu uso em projetos futuros. O elemento comparativo que se tinha, à época, era a "revisão de prontidão de voo", e a ideia de avaliar o "Nível de Maturidade Tecnológica" das novas tecnologias encontra-se materializada num relatório de 1969 e focada nos requisitos da tecnologia espacial (MANKINS, 2009).

Na década seguinte foi concretizado o desenvolvimento de uma escala de maturidade tecnológica, usando conceitos amplos de modo a abarcar as diversas tecnologias na mesma métrica, conforme aponta o National Aeronautics and Space Administration Office of Aeronautics and Space Technology (OAST). No final dos anos de 1970, uma escala foi desenvolvida por Stan Sadin que compreendia seis ou sete níveis, cada um com breves caracterizações (MANKINS, 2009; SEE et al., 2017).

Com a perda do ônibus espacial Challenger na década de 1980 ficou mais evidente a necessidade de integração de diversos programas e, consequentemente, de várias tecnologias, por isso, o sistema de TRL foi consolidado internamente pela NASA. Em julho de 1989, com a necessidade de comunicar o status da maturidade tecnológica entre os setores de planejamento e/ou de pesquisa, foi desenvolvido o modelo que se conhece hoje com os seus nove níveis (MANKINS, 2009; QUINTELLA; ROCHA, 2007).

Em resposta ao Augustine Report, o primeiro documento a utilizar a métrica do TRL foi o Integrated Technology Plan for the Civil Space Program (ITP), sendo também a primeira vez que uma organização cientifica utilizou o TRL para o gerenciamento de seus programas para comunicação externa. Em 1995, Mankins (1995) escreveu o seu White Paper, que influenciou o Departamento de Defesa dos EUA a adotar, em 2000, a métrica do TRL da NASA, também atendendo à recomendação feita, no ano anterior, pelo Departamento de Responsabilidade Geral dos Estados Unidos (MANKINS, 2009; COSTA; LEITE, 2018).

Assim, o sistema desenvolvido por Stan Sadin em 1974 foi aperfeiçoado em 1995 por Mankins, passando de sete para os atuais nove níveis. Nessa configuração atual, tem-se o nível TRL1 como o mais baixo e associado à pesquisa básica, e o nível TRL9 significa que a tecnologia está pronta para comercialização. Os níveis TRL1 e TRL2 estão relacionados com a pesquisa básica; TRL3 e TRL4, com a pesquisa para verificação de viabilidade; TRL3 a TRL6, com o desenvolvimento tecnológico; TRL5 a TRL7, com a demonstração da tecnologia; TRL6 a TRL8, com o desenvolvimento de sistemas e de subsistemas; TRL8 e TRL9 estão relacionados com os testes do sistema, lançamento e operação (QUINTELLA; ROCHA, 2007). 
Figura 1 - Termômetro de representação dos níveis de TRL

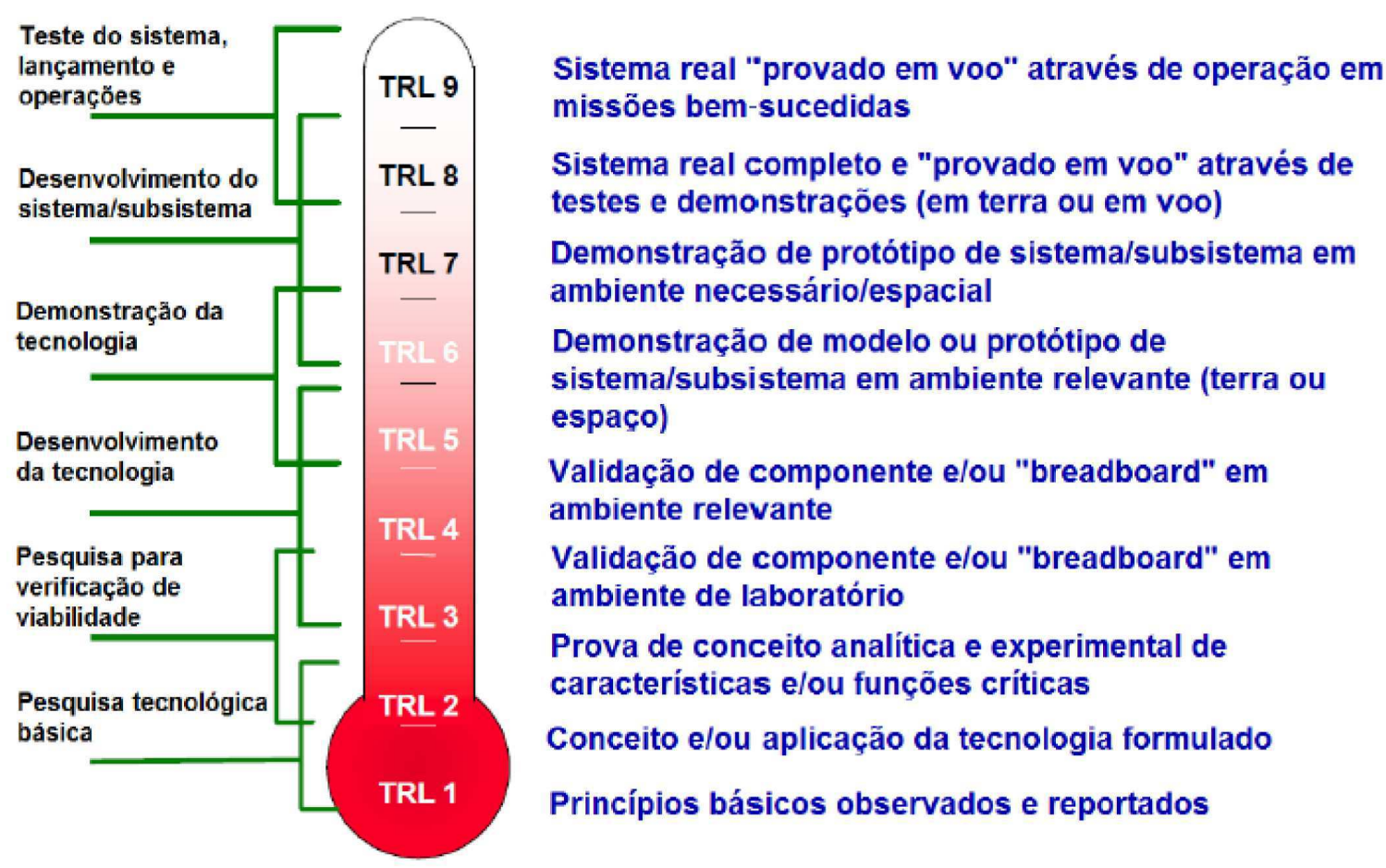

Fonte: Adaptada de Quintella et al. (2019) e da NASA (2014)

Ainda não há consenso na tradução do termo Readiness para o português, sendo utilizado o termo "prontidão" ou o termo "maturidade". Neste artigo, optou-se pelo termo "maturidade".

O nível de maturidade desenvolvido pela NASA está focado no segmento aeroespacial e nas necessidades internas da companhia. Outros setores também classificaram seus níveis de maturidade, baseando-se conceitualmente em TRL para o desenvolvimento de outras métricas mais específicas. Como citado por Bakke (2017), atualmente existem várias métricas/vários níveis que podem indicar o estágio de maturidade de uma tecnologia. Como exemplos, há: os Níveis de Maturidade da Fabricação (MRL); o Nível de Maturidade de Integração (IRL); o Nível de Maturidade de Design/Projeto; o Nível de Maturidade de Capacidade; o Nível de Maturidade de Software; o Nível de Maturidade Humana; o Nível de Maturidade de Logística; o Nível de Maturidade Operacional; o Nível de Maturidade de Inovação; e o Nível de Maturidade Programática.

De acordo com Straub (2015), a proposta para utilização do TRL10 foi inicialmente lançada por Brown e McCleskey, em 2000, e aprofundada em 2009, num artigo publicado por Robinson, que se focou na produção de transporte espacial. Esse TRL10 seria definido como Proven Operations, ou "Operações Comprovadas", e teria como objetivos verificar se a tecnologia foi usada sem incidentes, se foi certificada, se as falhas apresentadas são de conhecimento da equipe técnica e se o sistema opera em níveis aceitáveis, conforme manutenção programada. 
O modelo de nove níveis se encontra hoje amplamente difundido para diversas agências governamentais dos EUA: a Agência Espacial Europeia (ESA), o Departamento de Energia dos EUA (DoE), o Departamento de Segurança Interna (DHS) e o Departamento de Defesa. Outros setores tecnológicos também usam da TRL1 à TRL9, como o Setor Médico (Farmacêuticos, medicamentos); os setores Farmacêuticos (produtos biológicos, incluindo vacinas); os dispositivos médicos e a Tecnologia da Informação Médica e Informática Médica (IM); o setor de Energia; e o setor de Petróleo, Gás e Biocombustíveis.

No Brasil, algumas agências de fomento já utilizam o TRL para enquadramento dos projetos para financiamento, como a Embrapii e o Senai. Existe ainda a Norma ABNT ISO 162902013 - Sistemas espaciais - Definição dos níveis de maturidade da tecnologia (TRL) e de seus critérios de avaliação.

Este estudo pretende fazer uma análise comparativa exploratória para identificar os principais atores em conceitos de maturidade tecnológica, por meio de artigos e patentes, ou seja, identificar a difusão desse conhecimento. Seguidamente, foi mapeada a principal classificação de maturidade tecnológica, identificando os principais autores/titulares e suas evoluções anuais, além dos setores empresariais envolvidos.

\section{Metodologia}

Inicialmente, fez-se um levantamento preliminar nas bases de dados de artigos e patentes usando os termos Technology Readiness Levels e TRL, por serem os mais conhecidos quando se trata de Maturidade Tecnológica. Nesse levantamento, foram identificados o artigo de Bakke (2017) e o de Quintella e Rocha (2007), que fazem um levantamento das metodologias para avaliar a maturidade tecnológica.

Na sequência, foi realizado o comparativo dos resultados obtidos nas bases de patentes (Derwent, Questel Orbit, WIPO, Spacenet e Patent Inspiration) e de artigos (Web of Science, Scopus, Periódicos Capes e Google Academics). Os termos utilizados foram: Technology readiness levels; Manufacturing Readiness Levels; Programmatic Readiness Levels; Integration Readiness Levels; System Readiness Levels; Innovation Readiness Level; Technology Readiness Assessment; Design readiness level; Capability readiness level; Software Readiness level; Human readiness level; Logistics readiness level; Operational readiness level. Esses dados foram obtidos em agosto de 2018 e atualizados em agosto de 2019 (Figura 2), apresentando um aumento entre 19\% e 24\%. 
Figura 2 - Comparativo entre as bases de dados

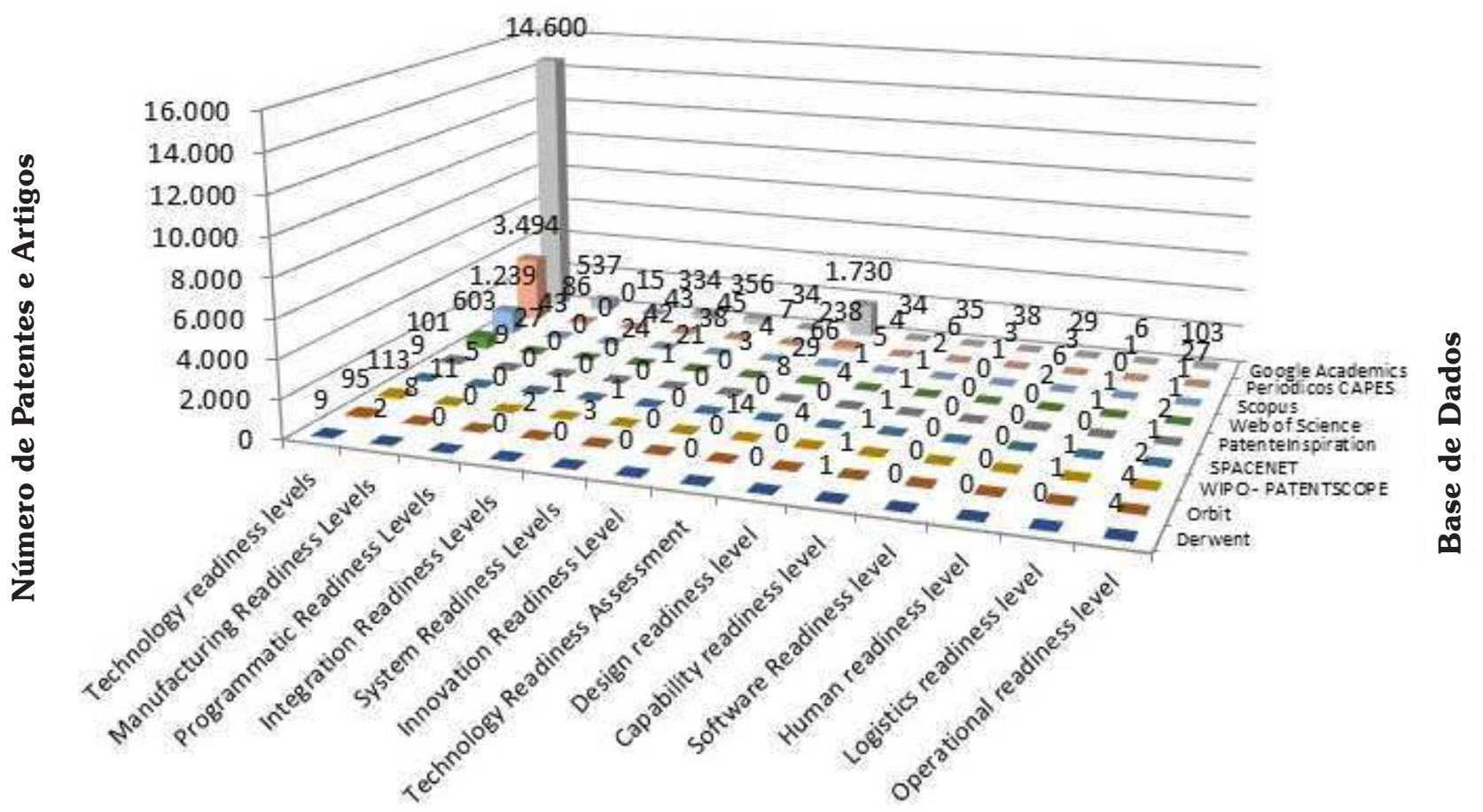

Palavras-chaves

Fonte: Elaborada pelos autores deste artigo (2019)

Diante dos dados apresentados na Figura 2, foram selecionados os melhores escopos e as melhores bases para a análise preliminar exploratória.

Para patentes, foi utilizado o Questel Orbit (ORBIT, 2018), já que ele permite o acesso à base worldwide da Espacenet, nesse caso, foram encontradas 432 famílias de patentes.

Para artigos, foi utilizada a base Scopus, pois o Google Academics e o(s) Periódicos Capes, apesar de apresentarem maior número de artigos, não dispõem de exportação adequada dos dados para a análise mais detalhada.

No Quadro 1 são apresentados as estratégias de busca e os respectivos resultados quantitativos. Como esperado, o número de artigos publicados (2.786) é muito superior ao número das patentes depositadas (110).

Quadro 1 - Estratégia de busca para formação das bases de dados a serem trabalhadas

\begin{tabular}{|c|c|c|c|c|c|}
\hline \multirow{2}{*}{ ID } & \multicolumn{2}{|c|}{ EstratÉGIA dE Busca } & \multicolumn{2}{|c|}{ Resultados } & \multirow[t]{2}{*}{$\begin{array}{c}\text { Nota } \\
\text { ExplicATIVA }\end{array}$} \\
\hline & SCOPUS (ARTIGOS) & ORBIT (Patentes) & SCOPUS & ORBIT & \\
\hline 1 & $\begin{array}{l}\text { TITLE-ABS-KEY ( "Technolog* } \\
\text { readiness leve*" ) }\end{array}$ & $\begin{array}{c}\text { (TECHNOLOG + } \\
\text { READINESS } \\
\text { LEVE }+ \text { )/TI/AB/TX }\end{array}$ & 1.240 & 95 & $\begin{array}{c}\text { Busca pelo } \\
\text { Termo Nível } \\
\text { de Maturidade } \\
\text { Tecnológica }\end{array}$ \\
\hline 2 & $\begin{array}{l}\text { TITLE-ABS-KEY ( "Manufactur* } \\
\text { Readiness Leve*" ) }\end{array}$ & $\begin{array}{c}\text { (MANUFACTUR }+ \\
\text { READINESS } \\
\text { LEVE }+ \text { )/TI/AB/TX }\end{array}$ & 43 & 8 & $\begin{array}{c}\text { Busca pelo } \\
\text { Termo Nível } \\
\text { de Maturidade } \\
\text { de Fabricação }\end{array}$ \\
\hline
\end{tabular}




\begin{tabular}{|c|c|c|c|c|c|}
\hline \multirow{2}{*}{ ID } & \multicolumn{2}{|l|}{ EstRATÉGIA dE BusCA } & \multicolumn{2}{|c|}{ Resultados } & \multirow[t]{2}{*}{$\begin{array}{c}\text { Nota } \\
\text { ExplicATIVA }\end{array}$} \\
\hline & SCOPUS (ARTIGOS) & ORBIT (Patentes) & SCOPUS & ORBIT & \\
\hline 3 & $\begin{array}{l}\text { TITLE-ABS-KEY ( "Programmatic* } \\
\text { Readiness Leve*" ) }\end{array}$ & $\begin{array}{c}\text { (PROGRAMMATIC }+ \\
\text { READINESS } \\
\text { LEVE }+ \text { )/TI/AB/TX }\end{array}$ & 0 & 0 & $\begin{array}{l}\text { Busca pelo } \\
\text { Termo Nível } \\
\text { de Maturidade } \\
\text { Programática }\end{array}$ \\
\hline 4 & $\begin{array}{l}\text { TITLE-ABS-KEY ( "Integrat* } \\
\text { Readiness Leve*" ) }\end{array}$ & $\begin{array}{c}\text { (INTEGRAT+ } \\
\text { READINESS } \\
\text { LEVE+)/TI/AB/TX }\end{array}$ & 42 & 2 & $\begin{array}{c}\text { Busca pelo } \\
\text { Termo Nível } \\
\text { de Maturidade } \\
\text { de Integração }\end{array}$ \\
\hline 5 & $\begin{array}{l}\text { TITLE-ABS-KEY ( "Syste* } \\
\text { Readiness Leve*") }\end{array}$ & $\begin{array}{c}\text { (SYSTE+ } \\
\text { READINESS } \\
\text { LEVE+)/TI/AB/TX }\end{array}$ & 38 & 3 & $\begin{array}{l}\text { Nível de } \\
\text { Maturidade } \\
\text { de Sistemas }\end{array}$ \\
\hline 6 & $\begin{array}{l}\text { TITLE-ABS-KEY ( "Innovat* } \\
\text { Readiness Leve*" ) }\end{array}$ & $\begin{array}{c}\text { (INNOVAT+ } \\
\text { READINESS } \\
\text { LEVE+)/TI/AB/TX }\end{array}$ & 4 & 0 & $\begin{array}{l}\text { Nível de } \\
\text { Maturidade } \\
\text { de Inovação }\end{array}$ \\
\hline 7 & $\begin{array}{l}\text { TITLE-ABS-KEY ( "Design* } \\
\text { readiness leve*" ) }\end{array}$ & $\begin{array}{c}\text { (DESIGN+ } \\
\text { READINESS } \\
\text { LEVE+)/TI/AB/TX }\end{array}$ & 5 & 0 & $\begin{array}{c}\text { Nível de } \\
\text { Maturidade de } \\
\text { Design/ Projeto }\end{array}$ \\
\hline 8 & $\begin{array}{l}\text { TITLE-ABS-KEY ("Capabilit* } \\
\text { readiness leve*") }\end{array}$ & $\begin{array}{c}\text { (CAPABILIT+ } \\
\text { READINESS } \\
\text { LEVE+)/TI/AB/TX }\end{array}$ & 2 & 1 & $\begin{array}{l}\text { Nível de } \\
\text { Maturidade de } \\
\text { Capacidade }\end{array}$ \\
\hline 9 & $\begin{array}{l}\text { TITLE-ABS-KEY ("Software* } \\
\text { Readiness leve*") }\end{array}$ & $\begin{array}{c}\text { (SOFTWARE+ } \\
\text { READINESS } \\
\text { LEVE+)/TI/AB/TX }\end{array}$ & 1 & 0 & $\begin{array}{l}\text { Nível de } \\
\text { Maturidade } \\
\text { de Software }\end{array}$ \\
\hline 10 & $\begin{array}{l}\text { TITLE-ABS-KEY ("Hum* } \\
\text { readiness leve*") }\end{array}$ & $\begin{array}{c}\text { (HUMAN+ } \\
\text { READINESS } \\
\text { LEVE+)/TI/AB/TX }\end{array}$ & 6 & 0 & $\begin{array}{l}\text { Nível de } \\
\text { Maturidade } \\
\text { dos recursos } \\
\text { humanos }\end{array}$ \\
\hline 11 & $\begin{array}{l}\text { TITLE-ABS-KEY ("Logistic** } \\
\text { readiness leve*") }\end{array}$ & $\begin{array}{c}\text { (LOGISTICS+ } \\
\text { READINESS } \\
\text { LEVE+)/TI/AB/TX }\end{array}$ & 0 & 0 & $\begin{array}{l}\text { Nível de } \\
\text { Maturidade } \\
\text { de Logística }\end{array}$ \\
\hline 12 & $\begin{array}{l}\text { TITLE-ABS-KEY ("Operationa* } \\
\text { readiness leve*") }\end{array}$ & $\begin{array}{l}\text { (OPERATION+ } \\
\text { READINESS } \\
\text { LEVE+)/TI/AB/TX }\end{array}$ & 1 & 5 & $\begin{array}{l}\text { Nível de } \\
\text { Maturidade } \\
\text { Operacional }\end{array}$ \\
\hline 13 & $\begin{array}{l}\text { TITLE-ABS-KEY ("Technolog* } \\
\text { Readiness Assessment*") }\end{array}$ & $\begin{array}{c}\text { (TECHNOLOG + } \\
\text { READINESS } \\
\text { ASSESSMENT +)/ } \\
\text { TI/AB/TX }\end{array}$ & 66 & 10 & $\begin{array}{l}\text { Avaliação de } \\
\text { Maturidade } \\
\text { Tecnológica }\end{array}$ \\
\hline 14 & 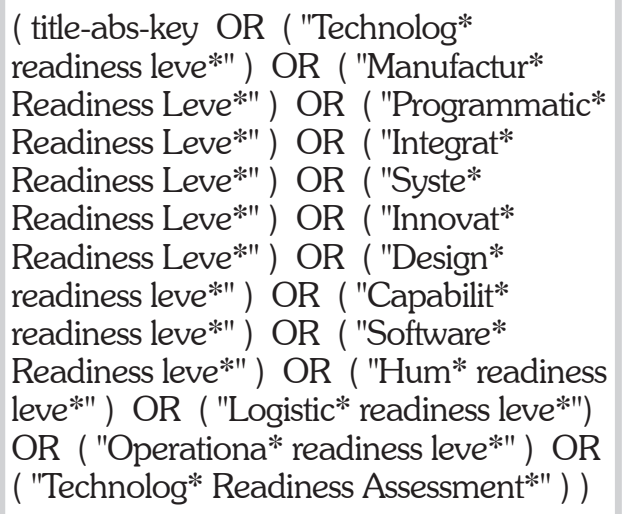 & $\begin{array}{l}\text { OR } 2 \text { OR } 3 \text { OR } 4 \\
\text { OR } 5 \text { OR } 6 \text { OR } 7 \text { OR } \\
8 \text { OR } 9 \text { OR } 10 \text { OR } \\
11 \text { OR } 12 \text { OR } 13\end{array}$ & 2.786 & 110 & $\begin{array}{l}\text { Pesquisa por } \\
\text { todos os termos } \\
\text { juntos que } \\
\text { servirá de base } \\
\text { para análise } \\
\text { dos dados. }\end{array}$ \\
\hline
\end{tabular}

Fonte: Elaborado pelos autores deste artigo (2019) 


\section{Resultados e Discussão}

O artigo Propulsive options for a manned mars transportation system, de Braun e Blersch (1991), foi o mais citado entre seus pares, com 21 menções. Um dos artigos, Technology readiness assessment of advanced space engine integrated controls and health monitoring, de Millis (1990), apesar de ser mais aderente a esta pesquisa, não teve nenhuma citação.

Em 1991, foram publicados cinco artigos, sendo que quatro deles têm como fonte a NASA e um deles a fonte é o Journal of Spacecraft and Rockets. Quando analisados por área, percebeu-se que quatro são de Engenharia e um é de Ciências da Terra e Planetárias, no entanto, todos versam sobre motores e sistema de propulsão aeroespacial e se encontram em fase de laboratório. Três dos artigos já fazem menção ao conceito atualmente utilizado de Maturidade Tecnológica. Um deles menciona que as tecnologias utilizadas se encontram em fase de laboratório, outro informa que se pretende chegar ao TRL6 em 2006, e o último aponta que, a depender do nível de maturidade, a tecnologia terá mais aceitabilidade que as demais.

A primeira patente a ser publicada (ZAVISKA OLDRICH; CHRISTIAN; HERBERT, 1996) que utilizou o termo Technology Readiness Level foi depositada pela Siemens, no ano de 1996, em mais de 10 países: Índia, Estados Unidos, Suíça, França, Reino Unido, Itália, Holanda, Suécia, Espanha, Alemanha, China, Japão, Rússia, Vietnã, República da Coreia e Taiwan, tendo expirado em 2016. A tecnologia refere-se ao método fuzzy, aplicado em centrais de geração termoelétrica. Essa metodologia teve sua primeira publicação em 1953, retornando em 1969. No ano da patente, esse método foi tema de 946 artigos publicados e, no acumulado até esta data, de 4.042 artigos.

O Gráfico 1 mostra o número de publicações anuais de artigos e de patentes. Percebe-se que há um aumento anual da publicação de artigos que versam sobre maturidade tecnológica, passando de 100 artigos por ano, a partir de 2008, e chegando a 374 em 2016.

Gráfico 1 - Número de publicações anuais de artigos e de patentes
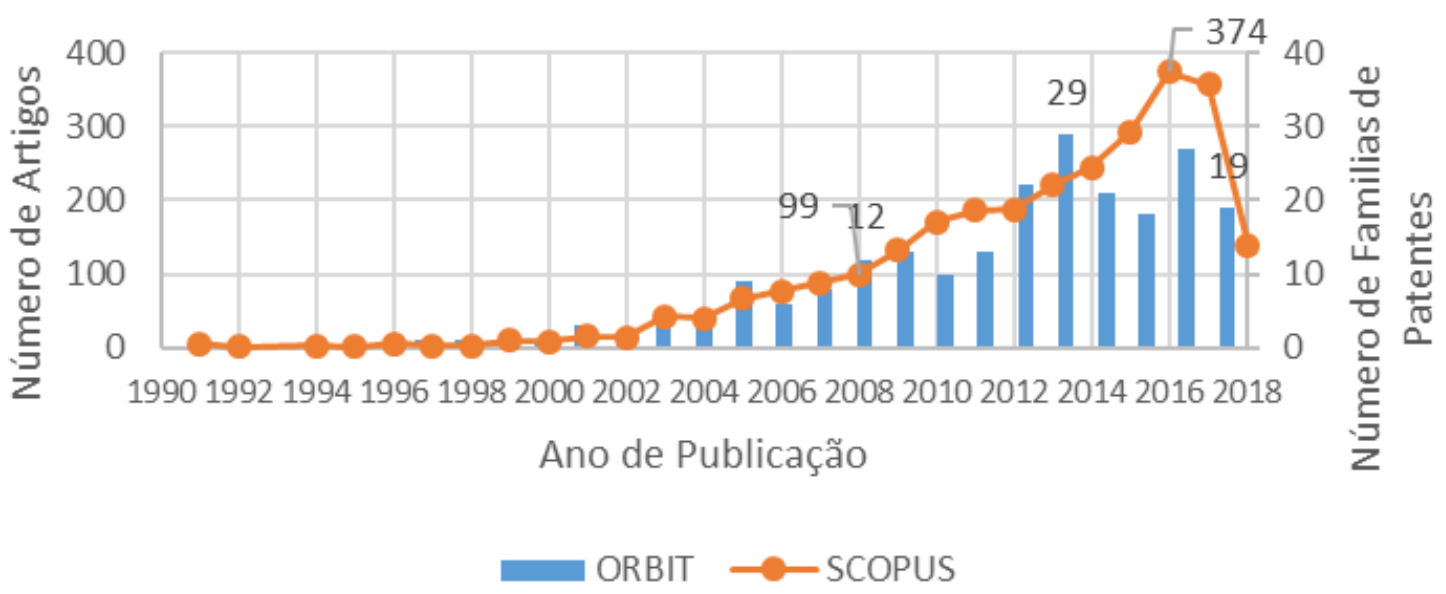

Fonte: Elaborado pelos autores deste artigo (2019)

Dois artigos, um de Sauser (2008) e outro de Rohloff (2009), chamam a atenção para a discussão sobre TRL, SRL e IRL, já que a utilização dessas três métricas auxiliam na redução de risco. Os demais artigos apenas citam TRL como forma de indicar qual o nível de maturidade da tecnologia está sendo tratado pelo artigo. 
Realizando a busca pelo termo TRL no título e em 2017 por considerar a evolução das métricas de TRL, identificou-se o artigo de Ma Kuan (2017), o autor junta todas as métricas para mensuração do nível de maturidade apresentada, construindo uma nova chamada de Maturidade Tecnológica Generalizada, e ainda propõe a criação de um aplicativo para avaliação e gerenciamento de tecnologias em projetos aeroespaciais. Essa tendência também é observada para as publicações de patentes, chegando a 29 famílias de patentes publicadas em 2014. Vale ressaltar que, em 2012, Ma Kuan já havia feito o depósito da patente de número 2012 CN-0411118 com o mesmo propósito do artigo publicado cinco anos mais tarde. Essa patente de 2012 foi reivindicada apenas na China e caducou em 2016. Isso pode indicar uma falta de mercado para a comercialização desse produto, haja vista que o órgão mais interessado, a NASA, foi quem desenvolveu todas as métricas de maturidade tecnológica. Ou foi uma falha na manutenção da Patente pela Beijing Institute of Information \& Control, que possui 12 patentes, porém, cinco delas já se encontram "mortas" (três revogadas e duas caducadas). Ao realizar uma análise por parceiros, por código da família e por data de depósito, não foi possível identificar nenhum motivo aparente que tivesse provocado essas situações.

Foram identificadas 223 organizações que publicaram pelo menos um artigo ou patente. Na base de dados de patentes, fez-se um corte nos 100 primeiros. Para identificação das empresas/universidades que submeteram patentes, fez-se o filtro por Assignee que identificou as 100 principais. Na base do Scopus, fez-se o filtro por Affiliation. Na sequência, foi realizado o cruzamento das empresas/universidades com artigos e patentes depositados.

Foram identificadas 11 organizações que detêm o maior número de patentes e de artigos publicados (Quadro 2). As organizações não empresariais têm maior número de artigos públicos, mas depositam poucas patentes, com exceção da NASA, que é a agência oficial do Governo Federal dos Estados Unidos para a pesquisa e desenvolvimento tecnológico e programas de exploração espacial (NASA, 2020). As empresas com maior número de patentes depositadas apresentam baixo nível de publicação de artigos. Algumas empresas, como a Boeing, a Thales e a Raytheon, apresentam uma relação de praticamente um artigo para uma patente.

Quadro 2 - Empresas/universidades com artigos e patentes citando maturidade tecnológica

\begin{tabular}{|c|c|c|c|c|c|}
\hline Empresa/ Universidade & Artigos & Patentes & $\begin{array}{c}\text { Número total } \\
\text { de Artigos }\end{array}$ & $\begin{array}{l}\text { Número total } \\
\text { de Patentes }\end{array}$ & \begin{tabular}{|c}
$\%$ de Patentes em \\
$\begin{array}{c}\text { Relação aos } \\
\text { Artigos }\end{array}$ \\
\end{tabular} \\
\hline NASA & 421 & 2 & 193.094 & 6.854 & $3,55 \%$ \\
\hline California Institute of Technology & 83 & 1 & 133.094 & 3.880 & $2,92 \%$ \\
\hline BOEING & 23 & 8 & 20.440 & 18.123 & $88,66^{\circ}$ \\
\hline Aerospace Corporation USA & 23 & 1 & 9.624 & 55.018 & $571,67 \%$ \\
\hline Thales Group & 11 & 1 & 13.050 & 6.931 & $53,11 \%$ \\
\hline SAFRAN (AIRCRAFT ENGINES) & 8 & 1 & 1.415 & 15.596 & $1102,19 \%$ \\
\hline Electric Power Research Institute & 7 & 1 & 8.019 & 27.586 & $344,01 \%$ \\
\hline Vrije Universiteit Brussel & 7 & 1 & 40.249 & 210 & $0,52 \%$ \\
\hline BEIJING INSTITUTE OF INFORMATION \& CONTROL & 6 & 2 & 1.540 & 12 & $0,78^{\circ}$ \\
\hline University of Texas at Austin & 6 & 1 & 146.353 & 40 & $0,03^{\circ}$ \\
\hline RAYTHEON & 4 & 4 & 12.457 & 12.980 & $104,20^{\circ}$ \\
\hline
\end{tabular}

Fonte: Elaborado pelos autores deste artigo

A Boeing é uma empresa do setor aeroespacial, com sede em Chicago, nos Estados Unidos, que tem faturamento anual de USD 101 trilhões em 2018 (INVESTING.COM, 2019) e investimento em inovação de 3 bilhões por ano, em diversos países. A partir de 2012, foi criado o 
Centro de Pesquisa e Tecnologia da Boeing no Brasil, em São José dos Campos, São Paulo. Essa empresa já tem parcerias firmadas com as seguintes instituições: DCTA, INPE, UFMG e USP, trata-se de um projeto de parcerias de longo prazo com outras universidades. No Brasil, as áreas de interesse para inovação encontram-se no setor de biocombustíveis sustentáveis para aviação, de gestão avançada de tráfego aéreo, de metais avançados e de biomateriais e de outras tecnologias importantes para a aviação (BOEING, 2008). A Boeing possui 18.948 depósitos de patentes (Quadro 2), sendo 10.022 concedidas, 7.232 "mortas" e 10 em litígio. $\mathrm{O}$ ano com maior número de patentes depositadas foi 2014, com 1.060. As principais áreas tecnológicas são Transporte (6.578), Mensuração (3.364), Tecnologia Computacional (2.312) e outras máquinas especiais (2.019). Os principais mercados são Estados Unidos (11.037), Organização Europeia de Patentes (4.662), China 2.959, Reino Unido (2.871), Japão (2.861) e Alemanha (2.397). O Brasil aparece em $9^{\circ}$ lugar, com 994 depósitos de patentes.

Thales é uma empresa francesa que comercializa sistemas de informação e serviços para as indústrias aeroespacial, de defesa e de segurança (THALES, 2020). Essa empresa está presente em 56 países e em 2018 teve um faturamento de 16.973 bilhões de euros (INVESTING. COM, 2019) e um investimento em inovação, pesquisa e desenvolvimento de 675 milhões de euros. Na área de inovação se destaca por ter se aproximado de 300 startups nos últimos três anos. Possui parceria institucional com 20 laboratórios de pesquisa; 50 acordos de parceria com universidades e institutos de pesquisa pública em diversos países; e tem 200 doutorandos apoiados em todo o mundo. Além disso, a empresa dispõe de áreas de interesse para inovação de conectividade, análise de dados, inteligência artificial, segurança cibernética, trens autônomos, sistemas não tribulados, aeronaves conectadas, estações espaciais não tripuladas. A Thales possui 5.168 patentes depositadas, 3.657 concedidas, 957 "mortas" e 554 pendentes. Em 2008, teve seu pico com 381 patentes depositadas, mas, nos anos subsequentes, se manteve no patamar de 300 por ano. As áreas de concentração são de Mensuração (1.857), Telecomunicações (1.254), Tecnologia Computacional (1.130) e Transporte (798). Os principais mercados são França (3.784), Organização Europeia de Patentes (2.345), Estados Unidos (2.007), Reino Unido (1.382) e Alemanha (1.326).

A Raytheon foi fundada em 1922 (RAYTHEON, 2019) e é um conglomerado norte-americano que atua na área de armamentos e de equipamentos eletrônicos para uso militar e comercial. Hoje, a Raytheon é líder em tecnologia e inovação especializada nos mercados de defesa, governo civil e segurança cibernética, em todo o mundo. Ela fornece eletrônica de última geração, integração de sistemas de mísseis e outros recursos nas áreas de sensoriamento; sistemas de comando, controle, comunicações e inteligência, além de segurança cibernética. Apresentou um faturamento no ano de 2017 de 27.058 bilhões de dólares (INVESTING.COM, 2019). Analisando as patentes, é possível perceber que a Raytheon possui um portfólio de 10.126 depósitos, sendo 3.908 concedidos, 5.854 "mortos", 364 pendentes e 21 em litígio. Teve seu pico em 2009, com 422 patentes/ano, mas nos anos subsequentes, esse número vem decrescendo, chegando, em 2016, a apenas 268 depósitos anuais. O domínio tecnológico está nas seguintes áreas: Mensuração (2.561), Telecomunicação (1.799) Tecnologia Computacional (1.232) e Máquinas Elétricas (1.153). Os principais mercados são Estados Unidos (4.035), Organização Europeia de Patentes (1.748), Reino Unido (1.006), Alemanha (986) e França (836). 
A Safran é um conglomerado francês de presença global, com mais de 58.000 funcionários em todo o mundo, que registrou vendas de 16,5 bilhões de euros em 2017 (SAFRAN, 2019). Composta de várias empresas, a Safran detém, sozinha ou em parceria, posições de liderança mundial ou europeia em seus mercados. Em fevereiro de 2018, a Safran assumiu o controle da Zodiac Aerospace, expandindo significativamente suas atividades de equipamentos de aeronaves. Com a Zodiac Aerospace, a Safran tem mais de 91.000 funcionários e teve um faturamento em 2018 de 21.025 bilhões de euros (INVESTING.COM, 2019). Em 2017, essa empresa investiu 1,4 bilhão de euros em pesquisa e desenvolvimento, possui um conselho científico composto de sete pesquisadores, internacionalmente reconhecidos, com o objetivo de fortalecer o vínculo com a comunidade científica. Registrou 850 patentes em 2017 e tem portfólio global de 30.000 patentes. A inovação está na cultura da empresa, que fomenta seus funcionários a proporem novas ideias. Em 2017, foram 112.500 ideias de melhoria implementadas que foram enviadas pelos funcionários. A Empresa enfatiza a inovação aberta, mantendo um canal por e-mail (saf. open-innovation@safran.fr), para que seus fornecedores/parceiros possam submeter/sugerir/ apresentar suas ideias. Em 2017, o Grupo firmou acordo de cooperação com o Senai Cimatec, na Bahia, para o desenvolvimento de gestão de ferramentaria na área aeronáutica, com uso de chips fornecidos pela instituição baiana. Com isso, surgiu a possibilidade de implantar no Estado da Bahia uma fábrica de eletrônicos (SOUZA, 2017). A Safran possui em seu nome 1.756 patentes e a Zodiac possui 387 patentes, perfazendo juntas um portfólio de 2.143 patentes, sendo 1.299 concedidas, 532 pendentes, 312 "mortas" e duas em litígio. De 1999 a 2004, essa empresa manteve uma média de 20 patentes/ano. A partir desse ano, esse número foi crescendo significativamente até chegar em 2016 a 284 depósitos. As principais áreas são Transporte (977), Motores, Bombas e Turbinas (690), Elementos Mecânicos (451) e Materiais Metalúrgicos (227). Os principais mercados são França (1.577), Organização Europeia de Patentes (1.386), Estados Unidos (1.188), Reino Unido (741), China (673) e Alemanha (657).

Como demonstrado pelo perfil das empresas, elas mantêm, de forma estruturada, parceria com instituição de pesquisa e possuem uma política clara de investimento em inovação. Esses dados são corroborados com as informações apresentadas no Quadro 2, que mostra sua atuação desde a pesquisa básica (artigos, TRL3), passando pelo desenvolvimento tecnológico (patentes, TRL4 e TRL5), até a colocação de suas inovações no mercado.

O Gráfico 2 mostra as áreas tecnológicas de artigos e patentes publicados. Os artigos que mais utilizaram um dos termos referentes à Maturidade Tecnológica estão na área de Engenharia (1.947), seguidos por Ciência da Computação (540), Física e Astronomia (477), Ciências da Terra e Planetárias (439), Energia (365) e Ciência de Materiais (356). Quando observadas as famílias das patentes, a ordenação é similar como demonstrada a seguir: G06 - Cômputo; Cálculo; Contagem/F - Processamento Elétrico de Dados Digitais (37); G06Q - Sistemas ou Métodos de Processamento de Dados especialmente adaptados para Propósitos Administrativos, Comerciais, Financeiros, de Gerenciamento, Supervisão ou Predição; Sistemas ou Métodos especialmente adaptados para Propósitos administrativos, comerciais, financeiros, de Gerenciamento, Supervisão ou Predição, não incluídos em outro Local (33); B64 - Aeronaves; Aviação; Cosmonáutica; B64G - Cosmonáutica; Veículos ou Equipamento para os mesmos (10); G06N - Sistemas de Computador Baseados em Modelos Computacionais Específicos (8); C - Química; Metalurgia; 07 - Química Orgânica; K - Peptídeos (6); H - Eletricidade; 04 - Técnica de Comunicação Elétrica; L - Transmissão de Informação Digital, por exemplo, Comunicação Telegráfica (5). 
Gráfico 2 - Áreas tecnológicas de artigos e patentes publicados
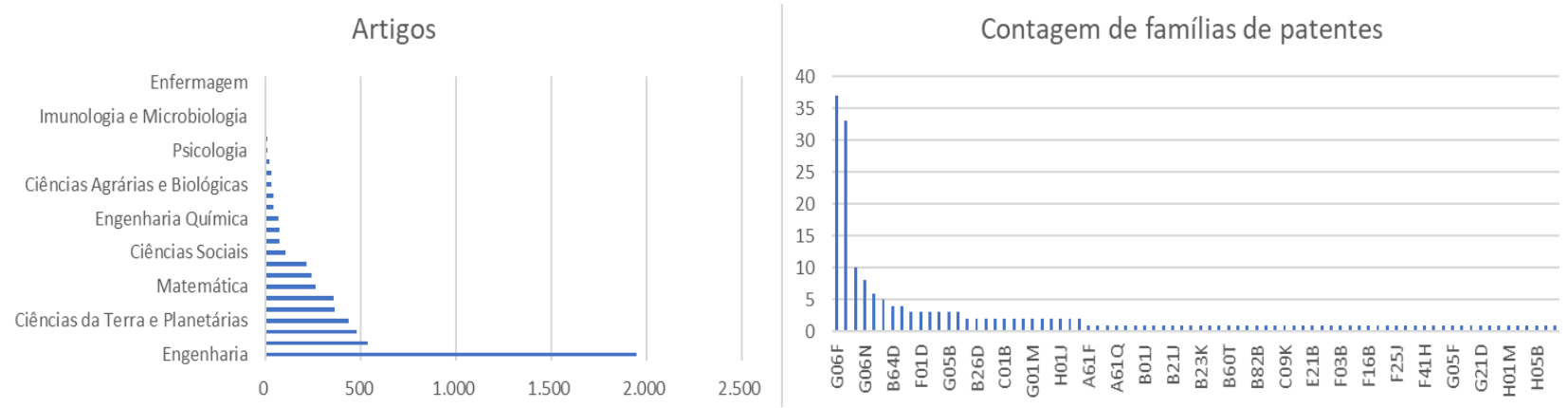

Fonte: Elaborado pelos autores deste artigo (2019)

Conforme já demonstrado no Quadro 2 e no descritivo das empresas, as áreas tecnológicas das patentes e dos artigos do Gráfico 2 estão alinhadas com as áreas de negócio das organizações.

O Quadro 3 mostra o recorte do passo 14 do Quadro 1 da estratégia de busca ao ser cruzado com o nome das empresas, assim, percebe-se um maior número de artigos na área de Engenharia (39), seguido da área de Física e Astronomia. As patentes estão concentradas na área de Informática (17), ambas utilizando os termos referentes à Maturidade Tecnológica.

Quadro 3 - Áreas Tecnológicas das empresas selecionadas

\begin{tabular}{|c|c|c|c|}
\hline $\begin{array}{c}\text { Áreas } \\
\text { TeCNOLÓGICAS }\end{array}$ & Artigos & Patentes & Áreas TeCnOlógicas \\
\hline Engenharia & 39 & 2 & $\begin{array}{l}\text { Engenharia Mecânica; Iluminação; Aquecimento; } \\
\text { Armas; Explosão (F41A - 1; F41H - 1). }\end{array}$ \\
\hline Física e Astronomia & 14 & 1 & $\begin{array}{c}\text { Transmissão De Informação Digital, por exemplo, } \\
\text { Comunicação Telegráfica (H04L -1). }\end{array}$ \\
\hline $\begin{array}{l}\text { Ciência de } \\
\text { materiais }\end{array}$ & 9 & 2 & $\begin{array}{l}\text { Investigação ou Análise dos Materiais pela Determinação de } \\
\text { suas Propriedades Químicas ou Físicas (G01N - 1); Trabalho } \\
\text { Mecânico com Pó Metálico; Fabricação de Artigos a partir } \\
\text { de Pó Metálico; Fabricação de Pó Metálico (Fabricação de } \\
\text { Ligas por Metalurgia do Pó C22c); Aparelhos ou Dispositivos } \\
\text { Especialmente Adaptados para Pó Metálico (B22F - 1). }\end{array}$ \\
\hline $\begin{array}{l}\text { Ciência da } \\
\text { Computação }\end{array}$ & 5 & 17 & $\begin{array}{l}\text { Cômputo; Cálculo; Contagem (G06F- } \\
\text { 10; G06Q - 6; G06N -1). }\end{array}$ \\
\hline
\end{tabular}

Fonte: Elaborado pelos autores deste artigo (2019)

A Figura 3 mostra a distribuição geográfica de origem de artigos e de patentes publicados. O principal país a citar em seus artigos as terminologias referentes à Maturidade Tecnológica é Estados Unidos (1.307), que também se configura como o principal depositário de patentes, o que é gerado pelo grande número de artigos publicados pela NASA. Para as patentes, as maiores titulares são as empresas estadunidenses Boeing e Raytheon. Outro fator que pode levar ao maior depósito de patentes nos EUA é sua legislação para o setor de Informática, já que permite apropriar patentes de software. O segundo maior player de patentes é a China, com 21 patentes, sendo o quarto em número de artigos (170). Outros importantes participantes na produção de artigos são o Reino Unido (337), Alemanha (269) e França (176). Já para patentes são: Canadá (9), Japão (7) Índia e Rússia (5). O Brasil aparece com duas patentes publicadas e 31 artigos (Figura 3). 
Figura 3 - Distribuição geográfica de origem de artigos e patentes publicados

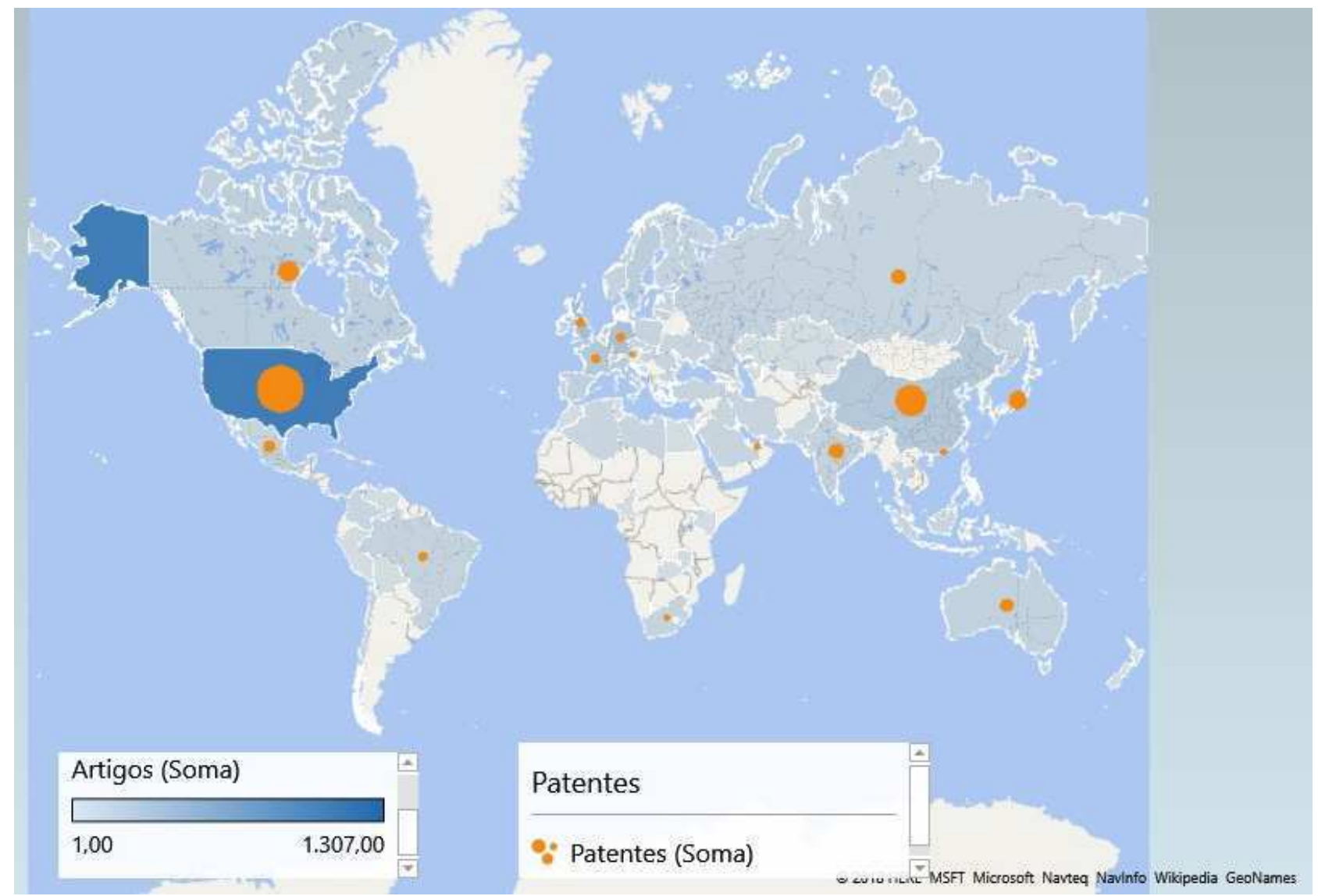

Fonte: Elaborada pelos autores deste artigo (2019)

\section{Considerações Finais}

O presente artigo teve como objetivo verificar os termos que são mais utilizados para se referir à Maturidade Tecnológica. Para tal, foram analisados artigos e identificados os seguintes termos: Technology readiness levels; Manufacturing Readiness Levels; Programmatic Readiness Levels; Integration Readiness Levels; System Readiness Levels; Innovation Readiness Level; Technology Readiness Assessment; Design readiness level; Capability readiness level; Software Readiness level; Human readiness level; Logistics readiness level; Operational readiness level.

Na linha do tempo, o termo Technology Readiness Levels foi cunhado pela NASA na década de 1970, e os primeiros artigos datam de 1991, mais de 20 anos depois. Nas patentes, os termos surgem em 1998, por meio de uma patente da Siemens. A partir de 2008, o número de publicações vem aumentando anualmente de forma significativa, tanto em patentes quanto em artigos.

Foi realizada uma pesquisa exploratória para verificar como artigos e patentes estão utilizando esses termos, selecionando a base Scopus e a base worldwide da Espacenet utilizando o Orbit. Foram identificados 2.787 artigos e 110 patentes.

O cruzamento das bases de dados mostrou que 11 empresas/universidades possuem patentes $e$ artigos publicados que fazem referência aos termos da pesquisa, destacando-se as empresas Boeing, Thales, Raytheon e Safran. Em pesquisa nos sites institucionais, verificou-se que essas empresas têm uma estrutura voltada para a inovação com investimentos na casa dos bilhões de 
dólares e parcerias com instituições de ensino e pesquisa, além disso, elas atuam praticamente no mesmo setor de segurança e aeroespacial. Vale ressaltar que as empresas/instituições que apresentam grande quantidade de artigos não possuem muitas patentes e vice-versa.

Quando foram analisadas as áreas de conhecimento, percebeu-se que os artigos têm maior número na área de Engenharia, seguidos da área de Ciência da Computação, o que tem forte correspondência com as patentes que estão concentradas em Processamento Elétrico de Dados (G06F), depois vem a área de Sistemas ou métodos de processamento de dados (G06Q). Essa tendência também aparece quando se faz o recorte para as empresas com maior número de publicações de famílias de patentes.

Quando se analisa por país, identifica-se que os Estados Unidos apresentam o maior número de artigos e de patentes. Em segundo lugar, aparece a China. A NASA é a organização com maior número de publicações de artigos, depois vem o Jet Propulsion Laboratory do California Institute of Technology, ambos estadunidenses. Em número de patentes se destacam também a Boeing e a MDA (Geospatial Services/ Information Systems), empresas canadenses que atuam na área aeroespacial.

\section{Referências}

BAKKE, Kjersti. Technology readiness levels use and understanding. 2017. Master (Thesis) University College South-East Norway, 2017. Disponível em: https://openarchive.usn.no/usn-xmlui/ handle/11250/2452831. Acesso em: 2 jan. 2018.

BOEING. Site institucional. [2008]. Disponível em: https://www.boeing.com/. Acesso em: 8 jul. 2018.

BRAUN, Robert D.; BLERSCH, Donald J. Propulsive options for a manned Mars transportation system. J. Spacecraft, [S.1.], v. 28, Issue 1, 1991. DOI: https://arc.aiaa.org/doi/10.2514/3.26213.

COSTA, Isabelle M. P.; LEITE, Handerson J. D. Nível de Maturidade Tecnológica (NMT) aplicado às tecnologias em saúde. In: BRASIL. Avanços, desafios e oportunidades no complexo industrial da saúde em serviços tecnológicos. Brasília, DF: Ministério da Saúde/Secretaria de Ciência, Tecnologia e Insumos Estratégicos/Departamento do Complexo Industrial e Inovação em Saúde, 2018. p. 186-200.

INVESTING.COM. Demonstração de Resultados Annual. [2019]. Disponível em: https:// br.investing.com/equities/. Acesso em: 21 jun. 2019.

MA KUAN, Wang Kunsheng. Generalized technology readiness level model. Teoria e Prática de Engenharia de Sistemas, [S.l.], v. 37, n. 3, p. 735-741, 2017. Disponível em: http:/www.sysengi. com/CN/10.12011/1000-6788(2017)03-0735-07. Acesso em: 17 ago. 2019.

MANKINS, John C. Technology readiness assessments: a retrospective. Acta Astronautica, [S.1.], n. 65. p. 1.216-1.223, 2009.

MANKINS, John C. Technology Readiness Levels. A White Paper, [S.1.], April 6, 1995. (Advanced Concepts Office. Office of Space Access and Technology NASA). 
MILLIS, M. Technology readiness assessment of advanced space engine integrated controls and health monitoring. The American Institute of Aeronautics and Astronautics, Inc. 1990. DOI: https://doi.org/10.2514/6.1991-3601.

NASA. Site institucional. [2020]. Disponível em: https://www.nasa.gov/about/index.html. Acesso em: 7 jul. 2020.

NASA. The TRL scale as a Research\&Innovation Policy Tool, EARTO Recommendations, 30 de abril de 2014, NASA, [2014]. Disponível em: https:/www.earto.eu/wp-content/uploads/The_TRL_ Scale_as_a_R_I_Policy_Tool_-_EARTO_Recommendations_-_Final.pdf. Acesso em: 7 jul. 2020.

ORBIT. [2018]. Disponível em: https://www.questel.com/business-intelligence-software/orbitintelligence/. Acesso em: 21 jun. 2018

QUINTELLA, Cristina M. et al. Maturidade Tecnológica: níveis de prontidão TRL. In: RIBEIRO, Núbia Moura. (org.). Prospecção Tecnológica. Salvador, BA: IFBA, 2019. v. 2. p. 18-59. 130p. Disponível em: http://www.profnit.org.br/pt/livros-profnit/. Acesso em: 21 jun. 2019.

QUINTELLA, Heitor Luiz Murat de Meirelles; ROCHA, Henrique Martins. Nível de maturidade e comparação dos PDPs de produtos automotivos. Produção, [S.l.], v. 17, n. 1, p. 199-217, jan.-abr. 2007. Disponível em: http://www.scielo.br/pdf/prod/v17n1/13.pdf. Acesso em: 21 jun. 2018.

RAYTHEON. Site institucional. [2019]. Disponível em: https:/www.raytheon.com/ourcompany/. Acesso em: 8 jul. 2020.

ROHLOFF, M. Case Study and Maturity Model for Business Process Management Implementation. Business Process Management, Proceedings of 7th International Conference, BPM 2009, Ulm, Germany, September 8-10, 2009. DOI: 10.1007/978-3-642-03848-8_10.

SAFRAN. Site institucional. [2019]. Disponível em: https://www.safran-group.com. Acesso em: 8 jul. 2018.

SAUSER, B. et al. A System Maturity Index for the Systems Engineering Life Cycle. Int. J. Industrial and Systems Engineering, [S.1.], v. 3, n. 6, 2008. DOI: 10.1504/IJISE.2008.020680.

SEE, Judi E. et al. Incorporating human readiness levels at Sandia National Laboratories. IEEE. Resilience Week (RWS), publicado em 2017. Disponível em: https://ieeexplore.ieee.org/ document/8088664. Acesso em: 12 ago. 2019.

SOUZA, Joyce. Jornal A Tarde. Matéria publicada em 9 de julho de 2017. Disponível em http:// atarde.uol.com.br/economia/noticias/1875958-safran-firma-acordo-com-cimatec-e-estuda-implantarfabrica-na-bahia. Acesso em: 8 jul. 2018.

STRAUB, Jeremy. In Search of Technology Readiness Level (TRL) 10. Aerospace Science and Technology, [S.l.], v. 46 p. 312-320, 2015. Disponível em: https://www.sciencedirect.com/science/ article/pii/S127096381500214X\#br0110. Acesso em: 17 ago. 2019.

THALES. Site institucional. [2020]. Disponível em: https://www.thalesgroup.com/en. Acesso em: 7 jul. 2020.

ZAVISKA OLDRICH, Z.; CHRISTIAN, F.; HERBERT, F. Method and device for rapid output adjustment of a power-generating facility. Titular: SIEMENS. Patente PCT WO97/18385. 1996. 


\section{Sobre os Autores}

\section{André Góes Paternostro}

E-mail: apater@hotmail.com Mestre em Propriedade Intelectual e Transferência de Tecnologia para Inovação (PROFNIT). Mestrado Profissional (www.profnit.org.br). Doutorado incompleto em Energia e Meio Ambiente pela Universidade Federal da Bahia. Mestrado incompleto em Regulação da Indústria de Energia pela Universidade Salvador (trancado) e Pós-Graduado no MBA em Relações Internacionais pelo Centro Universitário da Bahia.

Endereço profissional: CDM Group LTDA. Rua dos Radioamadores, n. 74, Pituaçu, Salvador, BA. CEP: 41741-080.

\section{Cristina M. Quintella}

E-mail: cris5000tina@gmail.com

Doutora em Ciências Moleculares pela University of Sussex, UK, em 1993. Pós-doutorados em Instituto Superior de Economia e Gestão da Universidade de Lisboa, Portugal, e no Instituto Politécnico de Setúbal, Portugal, além de diversas capacitações em Propriedade Intelectual e Transferência de Tecnologia (PI e TT) pela OMPI e pela INPI. Endereço profissional: Universidade Federal da Bahia, Instituto de Química, Departamento de Química Geral e Inorgânica, Campus de Ondina, Ondina, Salvador, BA. CEP: 40170-290.

\section{Handerson Jorge Dourado Leite}

E-mail: handerson@ifba.edu.br

Doutor em Saúde Pública, professor titular aposentado do Instituto Federal de Educação, Ciência e Tecnologia da Bahia (IFBA), foi Diretor de Ciência, Tecnologia e Inovação em Saúde da Secretaria de Saúde do Estado da Bahia, Diretor Geral do Polo de Inovação Salvador (Unidade especial do IFBA voltada para P, D \& I e serviços tecnológicos) e representante do IFBA na Rede Brasileira de Avaliação de Tecnologia em Saúde (REBRATS). Atualmente é Diretor de Inovação da Fundação de Amparo à Pesquisa do Estado da Bahia (FAPESB).

Endereço profissional: Instituto Federal da Bahia, Departamento de Tecnologia em Saúde e Biologia, Núcleo de Tecnologia em Saúde, Rua Emídio dos Santos, s/n, Barbalho, Salvador, BA. CEP: 40301-015. 